\title{
PRINCIPAL COMPONENT FILTER BANKS: EXISTENCE ISSUES, AND APPLICATION TO MODULATED FILTER BANKS
}

\author{
Sony Akkarakaran and P.P. Vaidyanathan \\ Department of Electrical Engineering 136-93 \\ California Institute of Technology \\ Pasadena, CA 91125 USA.
}

\begin{abstract}
Principal component filter banks (PCFB's) sequentially compress most of the input signal energy into the first few subbands, and are mathematically defined using the notion of majorization. In a series of recent works, we have exploited connections between majorization and convexity theory to provide a unified explanation of PCFB optimality for numerous signal processing problems, involving compression, noise suppression and progressive transmission. However PCFB's are known to exist for all input spectra only for three special classes of or thonormal filter banks (FB's): Any class of t wo c hannelFB's, the transform coder class and the unconstrained class. This paper uses the developed theory to describe techniques to examine existence of PCFB's. We prove that the classes of DFT and cosine-modulated FB's do not have PCFB's for large families of input spectra. This result is new and quite different from most known facts on nonexistence of PCFB's, which usually involve very specific examples and proofs with numerical optimizations.
\end{abstract}

\section{INTRODUCTION}

A generic filter bank (FB) based signal processing scheme is shown in Fig. 1. It uses a uniform $M$-channel FB and $M$ subband processors $P_{i}$, and is aimed at producing a certain desired signal $d(n)$ at the FB output. For example for signal compression, $d(n)$ is the FB input $x(n)$, and the processors $P_{i}$ are quantizers. For noise suppression, $x(n)=s(n)+\mu(n)$ where $\mu(n)$ is additive noise, and $d(n)$ is the pure signal $s(n)$. Here the $P_{i}$ could for instance be Wiener filters. We assume that $x(n)$ is a zero mean $\operatorname{CWSS}(M)$ random process (wide sense cyclostationary with period $M$ ) with a given second order statistics i.e. power spectral density (psd) matrix of its $M$-fold blocked version. The FB optimization problem involves finding the best FB from a given class $\mathcal{C}$ of uniform $M$-channel orthonormal FB's, for use in the system of Fig. 1.

The class $\mathcal{C}$ here could be for example the class $\mathcal{C}^{t}$ of transform coders (in which all filters have order $<M$ ), or that of FIR FB's (where all filters have order $<N$ for some fixed $N$ ), or the class $\mathcal{C}^{u}$ of unconstrained FB's (where the filters have no constraints besides those imposed by orthonormality). By 'best FB' we mean one that minimizes a

Work supported in part by the National Science Foundation under Grant MIP 0703755. well-defined objective on the class $\mathcal{C}$. This is usually the expected mean square value of the error $e(n)=d(n)-y(n)$ be$\mathrm{t}$ ween the desired and actual FB output. In many systems as in Fig. 1, the $P_{i}$ and the statistical assumptions on $d(n)$ and $x(n)$ are such that this error $e(n)$ is also $\operatorname{CWSS}(M)$. Equivalently, the subband errors $v_{i}^{(e)}(n)=v_{i}^{(d)}(n)-v_{i}^{(y)}(n)$ (errors between the true and desired outputs of the processors $P_{i}$ ) are jointly wide sense stationary (WSS). By FB orthonormality, the average mean square value of $e(n)$ is the average of the variances of the subband errors.

The best $\mathrm{FB}$ from the class $\mathcal{C}$ depends on $\mathcal{C}$, the input psd and the subband processors $P_{i}$ (i.e. the task for which the system is being used). In many situations it turns out to be the principal compnent $\mathrm{FB}$ (PCFB) for the class $\mathcal{C}$ and the given input psd. PCFB's will be reviewed in Section 2. The optimality of PCFB's for compression and progressive transmission has been observed to varying degrees in the past by a number of authors $[3],[2],[1],[6]$. More recently, we have shown [4] the precise connection between FB optimality and the principal component property: The PCFB is optimal whenever the minimization objective is a $c o n$ cave function of the vector of subband variances of the FB. This result explains many known optimality properties of PCFB's, and also proves their optimality for various noise suppression schemes, which had not been noticed earlier.

PCFB's are known to exist for all input spectra only for three special classes of FB's: Any class of t wo $\mathrm{c}$ hannel FB's, the transform coder class $\mathcal{C}^{t}$ and the unconstrained class $\mathcal{C}^{u}$. This paper shows techniques to examine existence of PCFB's. We prove that the classes of DFT and cosinemodulated FB's do not have PCFB's for large families of input spectra. This result is quite different from most known facts on nonexistence of PCFB's, which usually involve very specific examples and proofs with numerical optimizations.

\section{REVIEW OF PCFB OPTIMALITY}

This section briefly reviews the main results of $[4],[5]$. Definition: Majorization. Let $\mathbf{a}=\left(a_{0}, a_{1}, \ldots, a_{M-1}\right)^{T}$ and $\mathbf{b}=\left(b_{0}, b_{1}, \ldots, b_{M-1}\right)^{T}$ be t wo vectors in $\mathcal{R}^{M}$. Then $\mathbf{a}$ is defined to majorize $\mathbf{b}$ if the entries of these vectors, rearranged so that $a_{0} \geq a_{1} \geq \ldots \geq a_{M-1}$ and $b_{0} \geq b_{1} \geq$ $\ldots \geq b_{M-1}$, obey the property that

$$
\sum_{i=0}^{P} a_{i} \geq \sum_{i=0}^{P} b_{i} \quad \text { for all } P=0,1, \ldots, M-1,
$$


with equality holding when $P=M-1$. Evidently in this case, any permutation of $\mathbf{a}$ majorizes any permutation of $\mathbf{b}$.

Definition: PCFB's. Let $\mathcal{C}$ be the given class of orthonormal uniform $M$-channel FB's, and let $\mathbf{S}_{\mathbf{x x}}\left(e^{j \omega}\right)$ be the psd matrix of the $M$-fold blocked version of the FB input $x(n)$. $\mathrm{A} F \mathrm{FB}$ in $\mathcal{C}$ is said to be a principal component filter bank (PCFB) for the class $\mathcal{C}$ for the input psd $\mathbf{S}_{\mathbf{x x}}\left(e^{j \omega}\right)$, if its subband variance vector $\left(\sigma_{0}^{2}, \sigma_{1}^{2}, \ldots, \sigma_{M-1}^{2}\right)^{T}$ (where $\sigma_{i}^{2}$ is the variance of the subband signal $v_{i}^{(x)}(n)$ in Fig. 1) majorizes the subband variance vector of every $F B$ in the class $\mathcal{C}$. Alternatively, a PCFB may be defined as a FB minimizing over all FB's in $\mathcal{C}$, the mean-square error caused by dropping the $P$ weakest (lowest variance) subbands, for any $P=0,1, \ldots, M$. (Thus it is optimal for progressive transmission in this sense.) Note that in the definition (1) of majorization, the equality for $P=M-1$ is satisfied automatically for any $\mathbf{t}$ wo subband variance vectors $\mathbf{a}$ and $\mathbf{b}$, due to orthonormality of the FB.

Definition: Search-space. The optimization search-space is defined as the set $\mathcal{S}$ of all subband variance vectors corresponding to all FB's in the given class $\mathcal{C}$. So $\mathcal{S}$ is fully specified given $\mathcal{C}$ and the input psd matrix $\mathbf{S}_{\mathbf{x x}}\left(e^{j \omega}\right)$.

Theorem 1: PCFB's and polytopes. A PCFB for the class $\mathcal{C}$ for input psd $\mathbf{S}_{\mathbf{x x}}\left(e^{j \omega}\right)$ exists if and only if the convex hull $\operatorname{co}(\mathcal{S})$ of the search-space $\mathcal{S}$ is a polytope whose corners consist of all permutations of a single vector $\mathbf{v}_{*}$. Under this condition, $\mathbf{v}_{*}$ is the subband variance vector of the PCFB.

Theorem 2: Optimality of corners of polytopes. Let $f$ be a function whose domain $P$ is a convex polytope. If $f$ is concave on $P$, at least one of the corners of $P$ attains the minimum of $f$ over $P$. Further if $f$ is strictly concave, its minimum over $P$ has to be at a corner of $P$.

Theorem 3: Optimality of PCFB's. The PCFB for the class $\mathcal{C}$ (if it exists) is the optimum $\mathrm{FB}$ in $\mathcal{C}$ whenever the minimization objective is a concave function on the domain $\operatorname{co}(\mathcal{S})$. Further if this function is strictly concave, the optimum FB is necessarily a PCFB.

Recall that the convex hull of a set is the set of all possible convex combinations using elements of the set. A polytope is the convex hull of a finite set of points. The set of corners (or extreme points) of any compact convex set $D$ can be defined as the minimal subset of $D$ having $D$ as its convex hull. A polytope is a compact convex set with a finite number of corners. Theorem 3 follows directly from Theorems 1,2 which are proved in [4]. Theorem 3 shows PCFB optimality for numerous signal processing problems. Recall that with our problem formulation, the minimization objective is the average of the variances of the subband error signals $v_{i}^{(e)}(n)$ (Section 1$)$. These variances are often given by $E\left[\left|v_{i}^{(e)}(n)\right|^{2}\right]=h_{i}\left(\sigma_{i}^{2}\right)$, where $h_{i}$ is a function depending only on the subband processing (and not on the FB), and $\sigma_{i}^{2}$ is the subband signal variance. Thus the objective takes the form

$$
g\left(\sigma_{0}^{2}, \sigma_{1}^{2}, \ldots, \sigma_{M-1}^{2}\right)=\frac{1}{M} \sum_{i=0}^{M-1} h_{i}\left(\sigma_{i}^{2}\right),
$$

i.e. it is purely a function of the subband variance vector.
If in addition the $h_{i}$ are concave on $[0, \infty)$ then $g$ is concave on $\operatorname{co}(\mathcal{S})$. This happens in several problems involving compression and noise suppression, as explained in [4]. Thus for all these problems, a PCFB for the input is optimum.

\section{ON EXISTENCE OF PCFB'S}

For any class of 2-channel FB's, the PCFB is simply a FB maximizing the larger of its $t$ wo subband variances (thereby minimizing the other). For the class $\mathcal{C}^{t}$ of all $M$-channel orthogonal transform coders, the PCFB is the input KLT. This is by definition the unitary matrix $\mathbf{K}$ diagonalizing the autocorrelation matrix $\mathbf{R}$ of the $M$-fold blocked version of the FB input $x(n)$ in Fig. 1 (i.e such that $\mathbf{K R K}^{\dagger}$ is diagonal). For the class $\mathcal{C}^{u}$ of all (unconstrained) $M$-channel orthonormal FB's, the PCFB can be constructed by a procedure from [1]. It involves diagonalization of the psd matrix $\mathbf{S}_{\mathbf{x x}}\left(e^{j \omega}\right)$ (rather than $\mathbf{R}$ ) and an ordering of the resulting subband spectra at each frequency $\omega$. For both the classes $\mathcal{C}^{t}$ and $\mathcal{C}^{u}$, existence of the PCFB can basically be traced to the fact that the vector of eigenvalues of a Hermitian matrix majorizes the vector of its diagonal elements [9]. Thus for these classes, PCFB's exist for all input spectra.

Existence of a PCFB for a class $\mathcal{C}$ implies a very strong condition on the search-space $\mathcal{S}$ of all subband variance vectors of the FB's in $\mathcal{C}$. The convex hull $\operatorname{co}(\mathcal{S})$ has to be a polytope whose corners are all permutations of a single vector. It is possible to achieve this condition artificially by defining the class $\mathcal{C}$ to consist of all FB's producing subband variance vectors lying in such a polytope. The reason this is artificial is because the resulting class $\mathcal{C}$ depends on the input psd. Also, for very special input spectra it is possible that PCFB's exist for many more FB classes too, eg. for a white input, every $\mathrm{FB}$ in $\mathcal{C}$ is a $\mathrm{PCFB}$, no matter what the class $\mathcal{C}$. However it is quite plausible (though it has not been proved) that the only natural $\mathrm{FB}$ classes $\mathcal{C}$ for which PCFB's exist for all input psd's are $\mathcal{C}^{t}, \mathcal{C}^{u}$ and the 2-channel classes.

The basic method of proving nonexistence of PCFB's for a given class $\mathcal{C}$ is the following: If a PCFB exists, it simultaneously optimizes over $\mathcal{C}$, several functions of the subband variances. Thus a PCFB will not exist if we can find $t$ wo such functions that no single $\mathrm{FB}$ in $\mathcal{C}$ can simultaneously optimize. This method is used in [7],[8] for certain classes of FIR FB's, for a fixed input psd. The t wo functions used are the largest subband variance and the coding gain, both maximized by a PCFB if it exists. However all optimizations are numerical in [7],[8]. For the DFT and cosine-modulated classes, the functions we use are the largest and the least subband variances. The definition (1) of majorization shows that the PCFB maximizes the former and minimizes the latter.

\section{DFT AND COSINE-MODULATED CLASSES}

Definition. The class $\mathcal{C}^{d f t}$ of $M$-channel orthonormal DFT FB's is the one with all FB's as in Fig. 1 where the analysis filters are related by $H_{k}\left(e^{j \omega}\right)=P\left(e^{j\left(\omega-\frac{2 \pi k}{M}\right)}\right)$ for some filter $P\left(e^{j \omega}\right)$ called the prototype. For example, any $P\left(e^{j \omega}\right)$ which has an aliasfree $(M)$ support and has constant mag- 
nitude on its support (and is thus Nyquist $(M)$ ) produces a FB in $\mathcal{C}^{d f t}$.

Definition. The class $\mathcal{C}^{c m f b}$ of $M$-channel orthonormal cosine-modulated FB's (CMFB's) is the one containing all FB's as in Fig. 1 where

$$
H_{l}\left(e^{j \omega}\right)=P\left(e^{j\left(\omega-\frac{l \pi}{M}-\frac{\pi}{2 M}\right)}\right)+P\left(e^{j\left(\omega+\frac{l \pi}{M}+\frac{\pi}{2 M}\right)}\right)
$$

for some filter $P\left(e^{j \omega}\right)$ called the prototype. Any $P\left(e^{j \omega}\right)$ having an aliasfree $(2 M)$ support and with constant magnitude on its support, is a valid prototype.

Theorem 4: PCFB nonexistence for DFT class. There are families of input psd's such that the $M$-channel FB class $\mathcal{C}^{d f t}$ defined above $(M>2)$ does not have a PCFB.

Proof : Fig. 2 shows an input psd, t wo valid prototypes $P^{(j)}\left(e^{j \omega}\right)$, and the zeroth analysis filters $H_{0}^{(j)}\left(e^{j \omega}\right)=$ $P^{(j)}\left(e^{j \omega}\right), j=1,2$ in the DFT FB's corresponding to these prototypes. The value of the input psd at any point in the support of $H_{0}^{(1)}\left(e^{j \omega}\right)$ exceeds its value at any point outside this support. Thus $H_{0}^{(1)}\left(e^{j \omega}\right)$ maximizes its output variance among all Nyquist $(M)$ filters, i.e. it is the compaction filter [1] for the input psd. Since all filters in any $M$-channel orthonormal FB are Nyquist $(M)$, the filter $H_{0}^{(1)}\left(e^{j \omega}\right)$ produces the maximum subband variance achievable by any such FB, and hence by any FB in $\mathcal{C}^{d f t}$. Likewise, $H_{0}^{(2)}\left(e^{j \omega}\right)$ minimizes its output variance over all Nyquist $(M)$ filters. Thus it yields the minimum subband variance achievable by any orthonormal FB, and hence by any FB in $\mathcal{C}^{d f t}$. Now a PCFB simultaneously maximizes the largest and minimizes the least subband variance. Hence if a PCFB for $\mathcal{C}^{d f t}$ exists, it must contain both filters $H_{0}^{(j)}\left(e^{j \omega}\right), j=1,2$. This is impossible because these filters are not obtainable from each other by shift of an integer multiple of $\frac{2 \pi}{M}$, and hence a FB containing both of them cannot be in the class $\mathcal{C}^{d f t}$.

Theorem 5: PCFB nonexistence for the cosinemodulated class. There are families of input psd's such that the $M$-channel FB class $\mathcal{C}^{c m f b}$ defined above $(M>2)$ does not have a PCFB.

This is proved by exactly the same argument used for the class $\mathcal{C}^{d f t}$ (Theorem 4), for the input psd, prototypes $P^{(j)}\left(e^{j \omega}\right)$ and corresponding zeroth analysis filters $H_{0}^{(j)}\left(e^{j \omega}\right)$, $j=1,2$ shown in Fig. 3. The only difference is that we no longer have $H_{0}^{(j)}\left(e^{j \omega}\right)=P^{(j)}\left(e^{j \omega}\right)$. Also it takes more effort to show that no FB in $\mathcal{C}^{c m f b}$ can contain both filters $H_{0}^{(j)}\left(e^{j \omega}\right), j=1,2$. We do this by showing the following stronger result:

Result. A CMFB having $H_{0}^{(1)}\left(e^{j \omega}\right)$ of Fig. 3 as one of its filters is necessarily the CMFB produced by $P^{(1)}\left(e^{j \omega}\right)$ of Fig. 3 as prototype.

Proof : Define $Q\left(e^{j \omega}\right)=P\left(e^{j\left(\omega-\frac{\pi}{2 M}\right)}\right)$. Then from (3), the $l$-th filter $(0 \leq l \leq M-1)$ becomes

$$
H_{l}\left(e^{j \omega}\right)=Q\left(e^{j\left(\omega-\frac{l \pi}{M}\right)}\right)+Q\left(e^{j\left(\omega+\frac{(l+1) \pi}{M}\right)}\right)
$$

Now suppose the $k$-th filter is the ideal brickwall lowpass filter supported on $\left[-\frac{\pi}{M}, \frac{\pi}{M}\right]$, i.e.

$$
H_{k}\left(e^{j \omega}\right)=B\left(e^{j \omega}\right) \triangleq \begin{cases}s & \text { for } \omega \in\left[-\frac{\pi}{M}, \frac{\pi}{M}\right] \\ 0 & \text { for } \omega \in[-\pi, \pi] \backslash\left[-\frac{\pi}{M}, \frac{\pi}{M}\right]\end{cases}
$$

The problem is to examine the nature of the other filters $H_{l}\left(e^{j \omega}\right), l \neq k$. We do this by breaking the frequency band $[-\pi, \pi]$ into contiguous bands of length $\frac{\pi}{M}$. We will show that solutions $Q\left(e^{j \omega}\right)$ satisfying (5) are infinite in number. ${ }^{1}$ If $0<k<M-1$, none of these $Q\left(e^{j \omega}\right)$ are valid $C M F B$ prototypes. If $k=0$ or $k=M-1$, all of them are valid, and all generate the same $C M F B$.

To begin, note from (5) that $2 k+1$ and $M$ are coprime. To see this, equate the Fourier series coefficients of both sides of (5), using (4) for the left side. With $q_{n}$ denoting the Fourier series of $Q\left(e^{j \omega}\right)$, this yields

$$
q_{n} e^{j \frac{k \pi n}{M}}\left(1+e^{-j \frac{(2 k+1) \pi n}{M}}\right)=\frac{\sin \left(\frac{n \pi}{M}\right)}{n \pi}
$$

Suppose $2 k+1=n_{1} g$ and $M=n_{2} g$ for positive integers $n_{1}, n_{2}, g$. Then $n_{1}$ is odd, and the left side of $(6)$ is zero for $n=n_{2}$. But the right side is zero only if $n$ is a nonzero multiple of $M$. Hence $n_{2}$ is a multiple of $M$, and so $g=1$, i.e. $2 k+1$ and $M$ have no common factor. Next, define

$$
A_{i}\left(e^{j \omega}\right)=\left\{\begin{array}{cl}
Q\left(e^{j\left(\omega+\frac{i \pi}{M}\right)}\right) & \text { for } \omega \in\left[0, \frac{\pi}{M}\right] \\
0 & \text { for } \omega \in[-\pi, \pi] \backslash\left[0, \frac{\pi}{M}\right]
\end{array}\right.
$$

Observe $A_{i}=A_{i+2 M}$ for all integers $i$. Also, the $A_{i}$ fully determine the filters $H_{l}\left(e^{j \omega}\right)$, i.e. for $0 \leq l \leq M-1$,

$$
A_{i-l}\left(e^{j \omega}\right)+A_{i+l+1}\left(e^{j \omega}\right)=H_{l}\left(e^{j\left(\omega+\frac{i \pi}{M}\right)}\right), \quad \omega \in\left[0, \frac{\pi}{M}\right]
$$

We now use the known $H_{k}\left(e^{j \omega}\right)$ of (5) to attempt to solve for the $A_{i}$. Indeed, defining

$$
C\left(e^{j \omega}\right)= \begin{cases}s & \text { for } \omega \in\left[0, \frac{\pi}{M}\right] \\ 0 & \text { for } \omega \in[-\pi, \pi] \backslash\left[0, \frac{\pi}{M}\right]\end{cases}
$$

we see using (8) that (5) is equivalent to

$$
A_{i-k}+A_{i+k+1}=\left\{\begin{array}{cc}
C & \text { for } i \in\{0,1\} \quad(\bmod 2 M) \\
0 & \text { for all other } i
\end{array}\right.
$$

where we have dropped the argument $e^{j \omega}$ for convenience. Initializing $i=0$ and $A_{-k}=A+C$, we can solve the above system to find all $A_{i}$ uniquely in terms of $A$ and $C$. We do this by recursively writing $A_{i+k+1}$ in terms of $A_{i-k}$ and incrementing $i$ by $2 k+1$. The values are assigned in the sequence

$$
\begin{aligned}
\left(A_{-k}=A+C\right), & -A,+A,-A, \ldots,\left(A_{-1-k}=-A\right), \\
A+C, & -A-C, A+C, \ldots,\left(A_{-k}=A+C\right)(11)
\end{aligned}
$$

Since $2 k+1$ and $M$ are coprime, this process yields all $A_{i}$ and they satisfy (10) for all $i$. Since $2 k+1$ is odd,

$$
A_{i} \in\left\{\begin{array}{cl}
\{A, A+C\} & \text { for } i=-k \bmod 2 \\
\{-A,-A-C\} & \text { for } i=-1-k \bmod 2
\end{array}\right.
$$

Hence from (8) we see that the $H_{l}\left(e^{j \omega}\right)$ are in fact brickwall filters that are unaffected by $A$, and further

$$
H_{l}\left(e^{j \omega}\right)=\lambda_{i}^{l} \in\{0,+s,-s\} \text { for } \omega \in\left[\frac{i \pi}{M}, \frac{(i+1) \pi}{M}\right]
$$

\footnotetext{
${ }^{1}$ Nonuniqueness of $Q\left(e^{j \omega}\right)$ is easily seen: The filters $H_{l}\left(e^{j \omega}\right)$ of (4) do not c hange if $Q\left(e^{j \omega}\right)$ is replaced by $Q\left(e^{j \omega}\right)+Q^{\prime}\left(e^{j \omega M}\right)$ for any $Q^{\prime}\left(e^{j \omega}\right)$ satisfying $Q^{\prime}\left(e^{j \omega}\right)+Q^{\prime}\left(e^{j(\omega-\pi)}\right)=0$.
} 
Also, summing (10) over $i$ shows that $\sum_{i=0}^{2 M-1} A_{i}=C$, hence from (8) we see that $\sum_{i=0}^{2 M-1} \lambda_{i}^{l}=2 s$. Thus if $\lambda_{i}^{l}=-s$ for some $i$, then the total support of $H_{l}\left(e^{j \omega}\right)$ exceeds $\frac{2 \pi}{M}$, $\left|H_{l}\left(e^{j \omega}\right)\right|^{2}$ is not Nyquist $(M)$ and hence $H_{l}\left(e^{j \omega}\right)$ cannot be a filter in an orthonormal FB. So for the prototype to be valid, the $A_{i}$ must be such that $\lambda_{i}^{l} \neq-s$ for all $i, l$.

Now suppose $A_{i_{1}}=-A-C, A_{i_{2}}=A$ for $0 \leq i_{1}, i_{2} \leq$ $2 M-1$. Then from (12), $\left|i_{1}-i_{2}\right|$ is odd, so it equals $2 l+1$ for some integer $l$ such that $0 \leq l \leq M-1$. Then (8),(13) show that $\lambda_{\min \left(i_{1}, i_{2}\right)+l}^{l}=-s$. So either $A_{i} \neq-A-C$ for all $i$, or $A_{i_{1}}=-A-C$ and $A_{i} \neq A$ for all $i$. From the sequence (11), the former happens if and only if $(-1-k)+$ $(2 k+1)=-k \bmod 2 M$, i.e. $k=0$, while the latter happens iff $(-k)+(2 k+1)=-1-k \bmod 2 M$, i.e. $k=M-1$. We have already seen that $A$ does not affect the filters $H_{l}\left(e^{j \omega}\right)$. Now if $k=0$, we take $A=0$ in (11) and get $A_{0}=C, A_{1}=$ $\ldots=A_{2 M-1}=0$, which by definition (7) of $A_{i}$ means that $Q\left(e^{j \omega}\right)=C\left(e^{j \omega}\right)$. On the other hand if $k=M-1$, we take $A=-C$ and get $A_{M}=C, A_{0}=\ldots=A_{M-1}=$ $A_{M+1}=\ldots=A_{2 M-1}=0$, which means that $Q\left(e^{j \omega}\right)=$ $C\left(e^{j(\omega-\pi)}\right)$. Both these c hoices of $Q\left(e^{j \omega}\right)$ generate in t wo different orders, the same set of filters $H_{l}\left(e^{j \omega}\right)$.

\section{CONCLUDING REMARKS}

We have shown that the $M$-channel $(M>2)$ DFT and cosine-modulated FB classes do not have PCFB's for a large family of input spectra. The result is proved by choosing the spectra such that no single FB in the class can simultaneously maximize its largest and minimize its least subband variance. Unlike earlier proofs of PCFB nonexistence, this does not need any numerical optimizations. The nonexistence of PCFB for a class of FB's implies that no single FB in the class can simultaneously minimize all concave objectives. It also means that finding the optimum FB in the class for a given concave objective is likely to be analytically intractable.

\section{REFERENCES}

[1] P.P.Vaidyanathan, "Theory of Optimal Orthonormal Subband Coders," IEEE Trans. SP, vol. 46, no. 6, pp. 1528-1543, June 1998.

[2] M.K.Tsatsanis and G.B.Giannakis, "Principal Component Filter Banks for Optimal Multiresolution Analysis," IEEE Trans. SP, pp. 1766-1777, Aug. 1995.

[3] M.Unser, "An extension of the KLT for wavelets and perfect reconstruction filter banks," in Proc. SPIE no. 2034, Wavelet Appl. Signal Image Processing, San Diego, CA, 1993, pp.45-56.

[4] S.Akkarakaran and P.P.Vaidyanathan, "Filter Bank Optimization with Convex Objectives, and the Optimality of Principal Component Forms," submitted to IEEE Trans. Signal Proc essing

[5] S.Akkarakaran and P.P.Vaidyanathan, "On optimization of filter banks with denoising applications," in Proc. IEEE ISCAS, Orlando, FL, 1999.

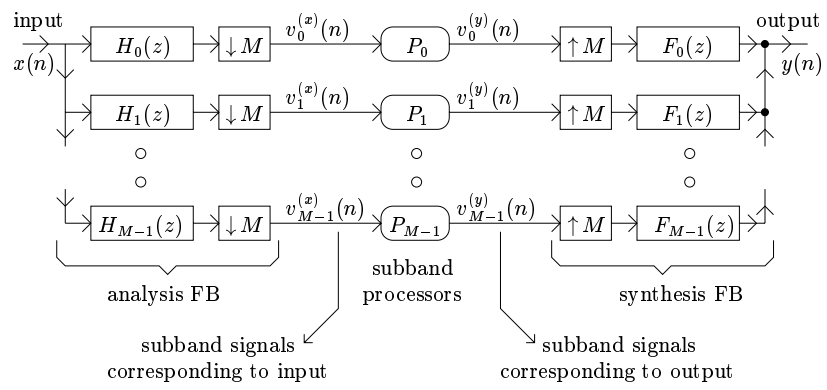

Figure 1: Generic FB based signal processing scheme.

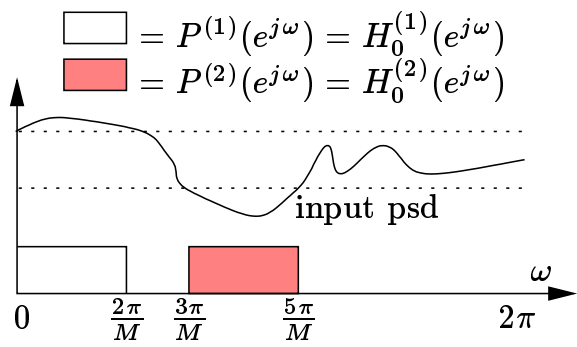

Figure 2: PCFB nonexistence: Class of DFT FB's.

[6] A.Kirac and P.P.Vaidyanathan, "Optimality of Orthonormal Transforms for Subband Coding," in Proc. IEEE DSP Workshop, Utah, August 1998.

[7] A.Kirac and P.P.Vaidyanathan, "On Existence of FIR Principal Component Filter Banks," in Proc. ICASSP, Seattle, May 1998.

[8] P.Moulin and M.K.Mihcak, "Theory and Design of Signal-Adapted FIR Paraunitary Filter Banks," IEEE Tr ans. SP, vol. 46, no. 4, pp. 920-929, April 1998.

[9] R.A.Horn and C.R.Johnson, Matrix Analysis. Cambridge University Press, 1985.

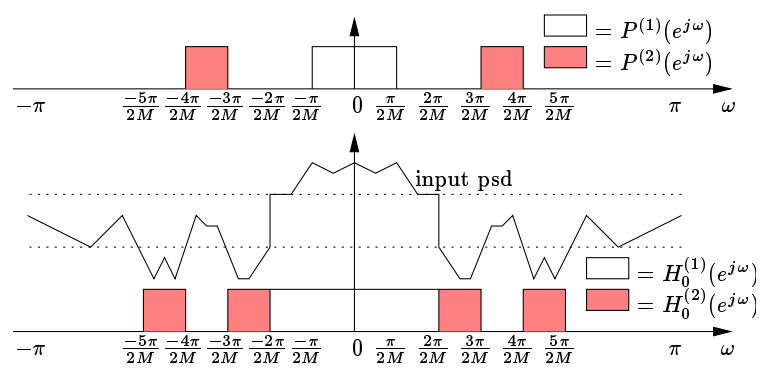

Figure 3: PCFB nonexistence: Cosine-modulated class. 